\title{
Metabolomic Analysis of Synovial Fluids from Rheumatoid Arthritis Patients Using Quasi-Targeted Liquid Chromatography-Mass Spectrometry/Mass Spectrometry
}

\author{
Hongxing Wang ${ }^{1}$, Kehua Fang ${ }^{2}$, Jibo Wang ${ }^{2}$, and Xiaotian Chang ${ }^{2}$ \\ ${ }^{1}$ Shandong University \\ ${ }^{2}$ Qingdao University
}

June 8, 2020

\begin{abstract}
Objective Synovial fluid (SF) accumulates extensively in joints of individuals with rheumatoid arthritis (RA), which reflects the pathological state of the synovium and disease activity. This study applied quasi-targeted liquid chromatography-mass spectrometry/mass spectrometry, an advanced metabolomics technique, to find characteristic metabolism in RA. Methods SF samples from the patients $(n=20)$ were collected and examined using the metabolomic technique. SF samples from patients with osteoarthritis (OA) $(n=20)$ were used as controls. Results Four hundred seventy nine variable metabolites were detected, and 250 of these metabolites were identified by searching the Human Metabolome Database (HMDB) and a self-constructed information list of possible metabolites. S-plot and volcano plot analysis detected 22 metabolites with differential levels in RA SF compared with those in OA SF. With these 22 candidate metabolites, pathway analysis using the Kyoto Encyclopedia of Genes and Genomes (KEGG) pathway database detected upregulation of pyrimidine metabolism and purine metabolism, and downregulation of fatty acid biosynthesis and unsaturated fatty acid biosynthesis in RA SF. Receiver operating characteristic (ROC) analysis and logistic regression models detected increased levels of guaiacol, naringenin, phenylpropanolamine and vanillylmandelic acid in RA SF. Furthermore, the naringenin level showed positive correlation with rheumatic factor (RF) and anti-cyclic citrillinated peptides (anti-CCP) levels. Conclusion Our study suggests disturbed pyrimidine metabolism, purine metabolism, fatty acid biosynthesis and unsaturated fatty acid biosynthesis as well as increased naringenin level are characteristic metabolism in RA.
\end{abstract}

\section{Hosted file}

20200607 S47 RA SF LCMS metabolism v7 to Clin Experimental Immun (combined).pdf available at https://authorea.com/users/331077/articles/457802-metabolomic-analysis-of-synovialfluids-from-rheumatoid-arthritis-patients-using-quasi-targeted-liquid-chromatographymass-spectrometry-mass-spectrometry 